\title{
Salvianolic Acid B Inhibited PPARY Expression and Attenuated Weight Gain in Mice with High-Fat Diet-Induced Obesity
}

\author{
Peijian Wang ${ }^{a}$ Shuyu Xub Wenzhang Lia Fang Wang ${ }^{a}$ Zhen Yang ${ }^{a} \quad$ Licheng Jiang $^{\mathrm{a}}$ \\ Qiulin Wang $^{\mathrm{a}}$ Mingqing Huang ${ }^{\mathrm{b}}$ Peng Zhou ${ }^{\mathrm{a}}$ \\ aDepartment of Cardiology, The First Affiliated Hospital, Chengdu Medical College, Chengdu, China, \\ ${ }^{b}$ College of Pharmacy, Fujian University of Traditional Chinese Medicine, Fuzhou, China
}

\section{Key Words}

Salvianolic acid B - Obesity - Adipose tissue - Peroxisome proliferator-activated receptor gamma $•$ CCAAT/enhancer binding protein $\alpha$

\begin{abstract}
Background/Aims: Obesity contributes to the development of cardiometabolic disorders such as type 2 diabetes, fatty liver disease and cardiovascular disease. Salvianolic acid B (Sal B) is a molecule derived from the root of Salvia miltiorrhiza (Danshen), which is a traditional Chinese medicine that is widely used to treat cardiovascular diseases. However, the role of Sal $B$ in obesity and obesity-related metabolic disorders is unknown. In this study, we aimed to investigate the effects of Sal B on high-fat diet-induced obesity and determine the possible mechanisms involved. Methods: Male C57BL/6J mice fed a high-fat diet for 12 weeks received a supplement of Sal B (100 mg/kg/day) by gavage for a further 8 weeks. These mice were compared to control mice fed an un-supplemented high-fat diet. 3T3-L1 preadipocytes were used in vitro studies. Results: Sal B administration significantly decreased body weight, white adipose tissue weight, adipocyte size and lipid (triglyceride and total cholesterol) levels in obese mice. Eight weeks of Sal B administration also improved the intraperitoneal glucose tolerance test (IPGTT) and intraperitoneal insulin tolerance test (IPITT) scores in high-fat diet-induced obese mice. In 3T3-L1 preadipocytes that were cultured in vitro and induced to differentiate, Sal $B$ reduced the accumulation of lipid droplets and lipid content in a dose-dependent manner. Immunoblotting indicated that Sal B decreased peroxisome proliferator-activated receptor gamma (PPAR $\gamma)$ and CCAAT/enhancer binding protein $\alpha(C / E B P \alpha)$ expression but increased the expression of GATA binding protein 2 and 3 (GATA 2, GATA 3) both in vivo and in vitro. Conclusion: Our data suggest that Sal B may reduce obesity and obesity-related metabolic disorders by suppressing adipogenesis. The effects of Sal B in adipose tissue may be related to its action on PPARY, C/EBP $\alpha$, GATA-2 and GATA-3.
\end{abstract}

P. Wang and S. Xu contributed equally to this work.

Mingqing Huang or Peijian Wang
College of Pharmacy, Fujian University of Traditional Chinese Medicine, No.1 Huatuo Road, Minhou Shangjie, Fuzhou, Fujian 350122 (PR China); or Department of Cardiology, The First Affiliated Hospital, Chengdu Medical College, Chengdu, Sichuan 610500 (PR China); E-Mail hmq1115@126.com or E-Mail wpjmed@aliyun.com 


\section{Introduction}

The prevalence of obesity is increasing worldwide. The reasons for this increase are complex and involve biological, behavioral and environmental factors [1]. Considering that obesity is deeply intertwined with both cardiovascular disease and diabetes, effective interventions are needed to reduce obesity rates on a population-wide basis [2]. Potential strategies for treating obesity include altering neural signals in the brain to regulate appetite, altering nutrient absorption in the gut, and modifying fat storage while promoting fat oxidation in adipose tissue [3]. Adipocyte differentiation plays a major role during the process of fat mass growth; therefore, control of adipogenesis may be a potential strategy for the prevention of obesity [4]. Adipogenesis is a complex process associated with coordinated changes in gene expression, cell morphology and hormone sensitivity [5]. These processes are influenced by several transcription factors, among which peroxisome proliferator-activated receptor gamma (PPAR $\gamma$ ) and CCAAT enhancer-binding proteins (C/ EBPs) have been extensively studied [5]. A cooperative interaction between PPAR $\gamma$ and C/ EBP $\alpha$ drives the expression of genes that are necessary for the generation and maintenance of the adipogenic phenotype; these genes induce morphological changes, lipid accumulation, and insulin sensitivity [6].

Salvianolic acid B (Sal B) is a water-soluble compound derived from the root of Salvia miltiorrhiza (Danshen), which is a Chinese medicinal herb. It has been used widely and successfully as a clinical therapy for various cardio-cerebrovascular disturbance-related diseases for hundreds of years in Asian countries [7, 8]. In recent years, in vivo and in vitro experiments have demonstrated that Sal B exerts a wide range of pharmacological effects. Sal B protects rat brains from ischemia and reperfusion injury by targeting the JAK2/STAT3 pathway [9] and inhibiting stromal cell-derived factor-1 alpha-stimulated cell proliferation and migration of vascular smooth muscle cells through suppression of the CXCR4 receptor [10]. Sal B also inhibits low-density lipoprotein oxidation and neointimal hyperplasia in endothelium-denuded hypercholesterolemic rabbits [11] and suppresses the maturation of human monocyte-derived dendritic cells associated with PPAR $\gamma$ [12]. These studies indicated that Sal B exerts protective effects in the cardio-cerebrovascular system. However, the role of Sal B in obesity is unknown.

Here, we used the high-fat diet-induced obese C57BL/6J mouse model and 3T3-L1 preadipocytes to explore the anti-obesity properties of Sal B. Our results indicated that Sal B treatment reduced weight gain, hyperlipidemia and insulin resistance in mice with high-fat diet-induced obesity. We also showed that Sal B suppressed the expression of PPAR $\gamma$ and C/ EBP $\alpha$ both in vivo and in vitro. Thus, Sal B may be effective in the treatment of obesity.

\section{Materials and Methods}

\section{Animal treatment}

Eight-week-old male C57BL/6J mice (Jackson Labs, Bar Harbor, ME) were given a high-fat diet (SLAC Laboratory Animal Co., Ltd., Shanghai, China) for 12 weeks. Sal B (100 mg/kg daily, Sal B group, n=10) or saline (control group, $\mathrm{n}=10$ ) was then given by oral gavage for an additional 8 weeks. The composition of the high-fat diet was (wt/wt): $32.06 \%$ lard, $3.27 \%$ soybean oil, $26.17 \%$ casein, $16.35 \%$ maltodextrin, 9.0\% sucrose, $6.54 \%$ cellulose, $4.58 \%$ AIN-93 minerals, $1.31 \%$ AIN-93 vitamins, $0.39 \%$ L-cystine and $0.33 \%$ choline. All the animals were maintained under controlled temperature $\left(22 \pm 2^{\circ} \mathrm{C}\right)$ and lighting (lights on at 6:00 $\mathrm{AM}$ and off at 6:00 PM) conditions. The investigation conformed to the Guide for the Care and Use of Laboratory Animals that was published by the US National Institutes of Health (NIH Publication No. 8523, revised 1996) and was approved by the Experimental Animal Ethics Committee of Fujian University of Traditional Chinese Medicine. Sal B was purchased from Shanghai Tauto Biotech Co., Ltd. (Shanghai, China) and was analyzed by high-performance liquid chromatography. The purity of Sal B was greater than $98 \%$. 
Intraperitoneal glucose tolerance and insulin tolerance tests

Intraperitoneal glucose tolerance tests (IPGTT) and intraperitoneal insulin tolerance tests (IPITT) were performed as previously described [13]. The IPGTT was performed after an overnight fast (14 h). Glucose ( $2 \mathrm{~g} / \mathrm{kg}$ body weight) was administered via injection into the peritoneal cavity, and blood was obtained from the tail at 0, 30, 60 and $120 \mathrm{~min}$ after glucose administration. Blood glucose levels were determined using the OneTouch Ultra blood glucose meter (LifeScan, CA, USA). The IPITT was performed using fed mice on a different day. Humulin R ( $0.75 \mathrm{U} / \mathrm{kg}$ body weight) (Eli Lilly and Co., IN, USA) in sterile saline was administered via injection into the peritoneal cavity. Glucose levels in tail blood were determined at $0,15,30,45$ and 60 min after insulin injection.

\section{Histological analysis}

Adipose tissue was isolated from mice, fixed in $10 \%$ formalin, and embedded in paraffin. Sections (10 $\mu \mathrm{m}$ thickness) were obtained and later stained with hematoxylin and eosin (H\&E) for the histological examination of adipocytes. Tissue sections were observed with a microscope (Nikon, TE2000) [14].

\section{Analysis of lipids and insulin levels}

Heparinized blood was drawn from the abdominal aorta of anesthetized mice. Concentrations of plasma lipids (triglyceride and total cholesterol) and insulin levels were determined using an enzymatic assay according to the manufacturer's instructions (Applygen Technologies Inc., China). Triglycerides were extracted from adipocytes and measured using an enzymatic colorimetric assay kit according to the manufacturer's instructions (Applygen Technologies Inc., China).

\section{Culture of 3T3-L1 preadipocytes and stimulation}

3T3-L1 preadipocytes were acquired from the China Center for Type Culture Collection (CCTCC, Wuhan, China). The cells were grown in Dulbecco's modified Eagle's medium (DMEM; GIBCO-BRL, Grand Island, NY, USA) containing 10\% fetal bovine serum (FBS), $100 \mathrm{U} / \mathrm{mL}$ penicillin, $100 \mu \mathrm{g} / \mathrm{mL}$ streptomycin and $2 \mathrm{mmol} / \mathrm{L}$ glutamine in an atmosphere of $5 \% \mathrm{CO}_{2}$ at $37^{\circ} \mathrm{C}$ [15]. Once confluence was reached, the cells were exposed to a pro-differentiation regimen including dexamethasone $(1 \mu \mathrm{M})$, insulin $(5 \mu \mathrm{g} / \mathrm{mL})$, and isobutylmethylxanthine $(0.5 \mathrm{mM})$. After 2 days, the cells were then maintained in media containing insulin until they were ready for harvest at day 7 [15]. Mature, differentiated 3T3-L1 cells were fixed and stained with the lipophilic dye oil red 0 (Sigma-Aldrich). Red staining reveals lipid droplets in the cytoplasm, indicating adipocyte differentiation.

Differentiation was also induced in the absence or presence of Sal B $(10 \mathrm{nM}, 100 \mathrm{nM}, 1 \mu \mathrm{M}, 10 \mu \mathrm{M}$, and $100 \mu \mathrm{M})$. Sal B was continuously present during the cell culture if not otherwise indicated.

\section{Western blot analysis}

Immunoblotting for PPAR $\gamma, \mathrm{C} / \mathrm{EBP} \alpha, \mathrm{GATA}$ binding protein 2 (GATA 2), GATA binding protein 3 (GATA 3) and GAPDH was performed as previously described [16]. Primary antibodies from Santa Cruz Biotechnology (Santa Cruz, CA, USA) were used for white fat tissue and mature 3T3-L1 cells. After incubation with the secondary antibodies for $1 \mathrm{~h}$, the proteins were detected by enhanced chemiluminescence and quantified using a Gel Doc 2000 Imager (Bio-Rad). The immunoblots showed bands of the expected size for PPAR $\gamma, \mathrm{C} /$ $\mathrm{EBP} \alpha, \mathrm{GATA} 2$, and GATA 3. Each sample was processed three times.

\section{Statistics}

The results are expressed as the mean \pm SEM. Comparisons between groups were analyzed using oneway analysis of variance with Bonferroni's multiple comparison post hoc tests or Student's $t$ test where appropriate. Two-sided P values $<0.05$ were considered statistically significant.

\section{Results}

Sal B reduced weight gain and hyperlipidemia in high-fat diet-induced obese mice

Sal B (100 mg/kg daily) was added after a high-fat diet was administered for 12 weeks. Sal B did not affect the food intake of the mice (Fig. 1A). As shown in Figure 1B and 1C, mice 
Fig. 1. Effects of salvianolic acid $\mathrm{B}$ on weight gain and lipid levels in high-fat diet-induced obese mice. A: Daily food intake during the first 14 days after the start of treatment in mice $(n=10)$. B: Body weights of mice fed a high-fat diet (HD) and mice fed a high-fat diet and treated with salvianolic acid B (Sal B) $(\mathrm{HD}+\mathrm{Sal} B)(\mathrm{n}=10)$. C: Sal B attenuates the increase in circumference of high-fat dietinduced obese mice $(n=10)$. D and E: Effects of Sal B on plasma levels of triglyceride (TG) and total cholesterol (TC) in highfat diet-induced obese mice $(\mathrm{n}=10) . \quad \# P<0.05, \quad \# \# P<0.01$ versus the HD group. The data are represented as the mean \pm SEM and were analyzed with Student's unpaired t-test.
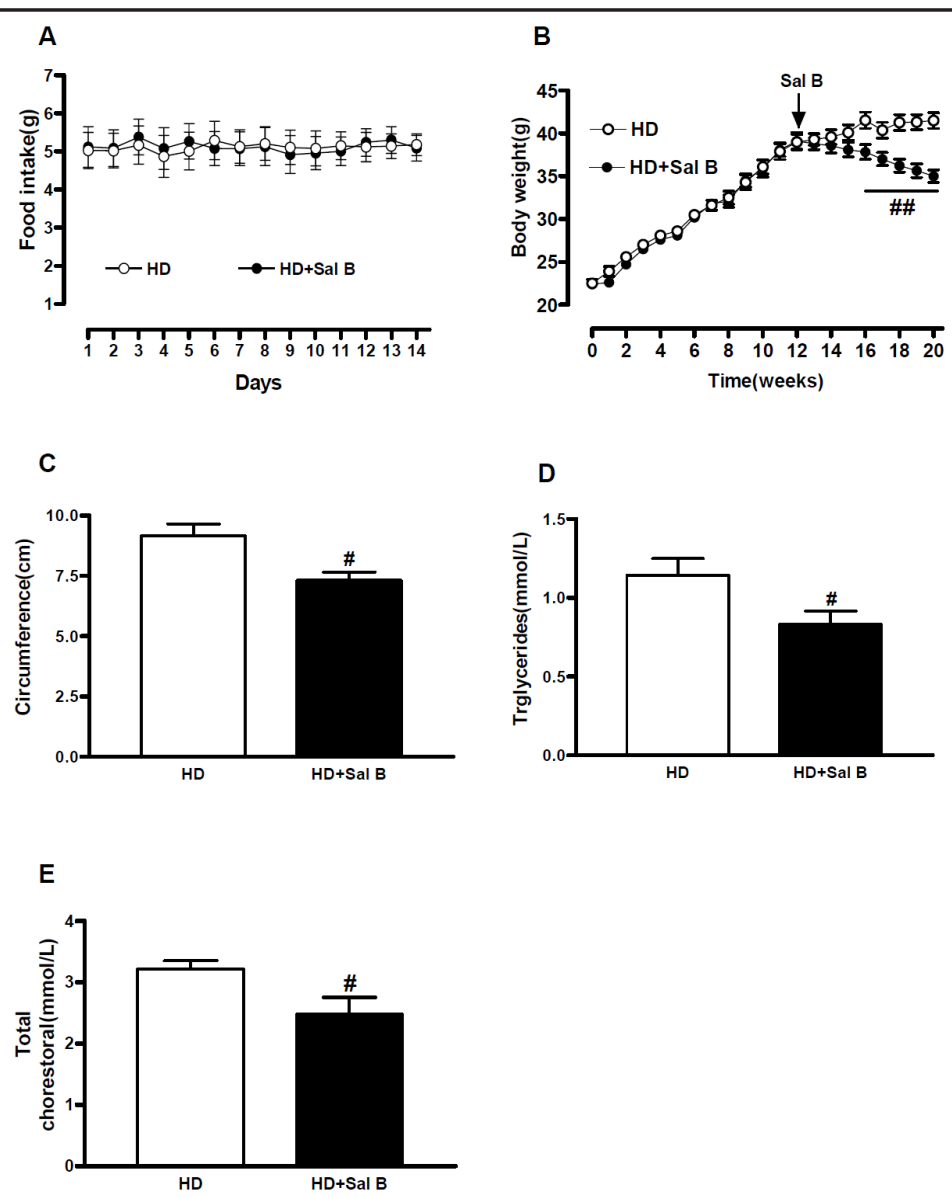

treated with Sal B for 8 weeks exhibited significantly reduced body weight and circumference despite the continuous administration of a high-fat diet. The weight gain in the high-fat diettreated group was $41.51 \pm 0.93 \mathrm{~g}$, while the weight gain in high fat diet plus Sal B group was $35.01 \pm 0.75 \mathrm{~g}(\mathrm{n}=10)$. After 8 weeks of Sal B administration, the mice were sacrificed and the plasma lipid levels were analyzed. Eight weeks of Sal B administration decreased both the triglyceride (TG) and total cholesterol (TC) levels in high-fat diet-induced obese mice (Fig. $1 \mathrm{D}$ and $\mathrm{E})$.

Sal $B$ reduced the weight of white adipose tissue and adipocyte size in high-fat dietinduced obese mice

We next investigated the effects of Sal B on white adipose tissue weight and adipocyte size in high-fat diet-induced obese mice. Eight weeks of Sal B treatment reduced the weight of both visceral adipose tissue and subcutaneous adipose tissue (Fig. 2A and B). Histological analysis showed that adipocyte size in both visceral adipose tissue and subcutaneous adipose tissue was smaller in the Sal B group than in the control group fed a high-fat diet (Fig. 2C, D and E).

Effects of Sal B on glucose metabolism and insulin resistance in high-fat diet-induced obese mice

It has been demonstrated that obesity, and perhaps central obesity, promotes insulin resistance [17]. To test whether Sal B could improve obesity-induced insulin resistance, we compared fasting glucose levels, the degree of glucose intolerance and insulin sensitivity in the two groups of mice. Fasting glucose and insulin levels were lower in the obese mice treated with Sal B than in the control mice (Fig. 3A and B). IPGTTs and IPITTs revealed that the obese mice were glucose intolerant and insulin resistant. Furthermore, as shown in Figure 
Fig. 2. Effects of salvianolic acid $B$ on the weight of adipose tissue and size of adipocytes in highfat diet-induced obese mice. A and B: Effects of Sal B on white adipose tissue (WAT) in visceral and subcutaneous fat in high-fat diet-induced obese mice $(n=10)$. C and D: Histology of visceral and subcutaneous adipose tissue $(n=6)$. E and F: Adipocyte size in visceral WAT and subcutaneous WAT (sub-WAT). The scale bar represents $50 \mu \mathrm{M}$. $\# P<0.05$, \#\#P<0.01 versus the HD group $(n=6)$. The data are represented as the mean \pm SEM and were analyzed with Student's unpaired t-test.

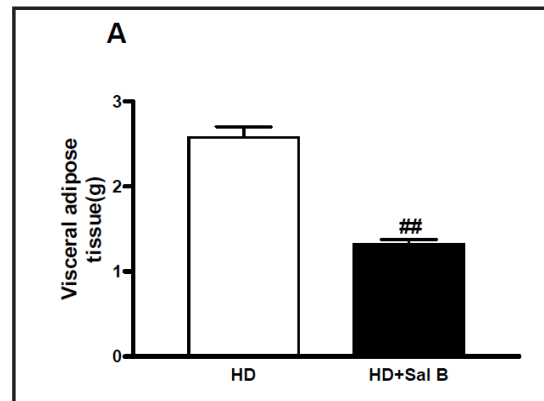

B
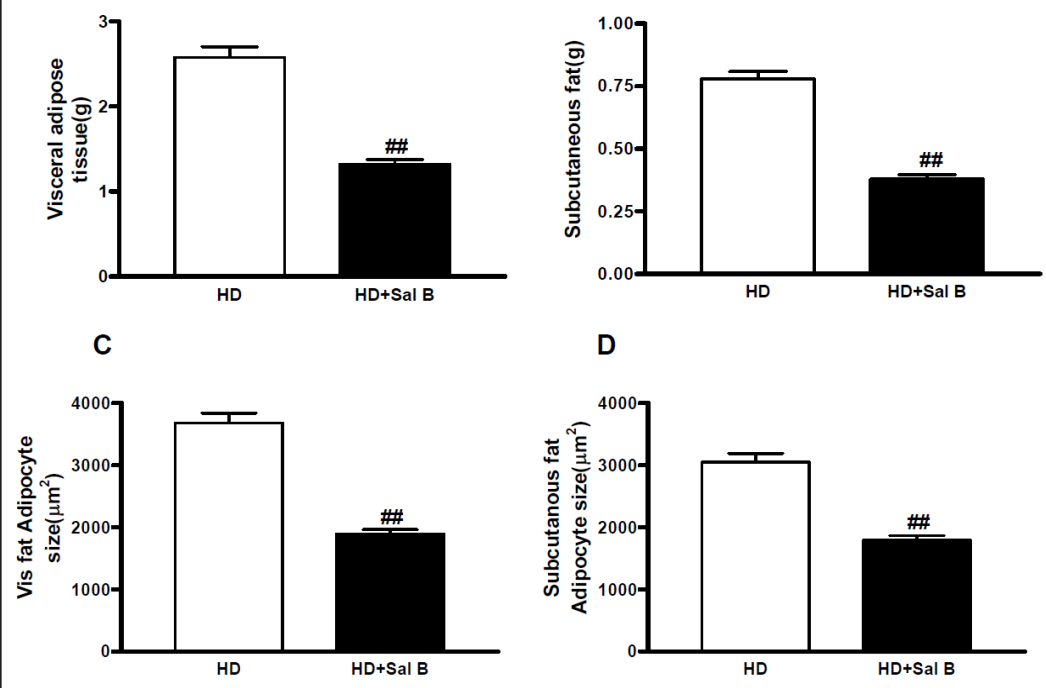

D

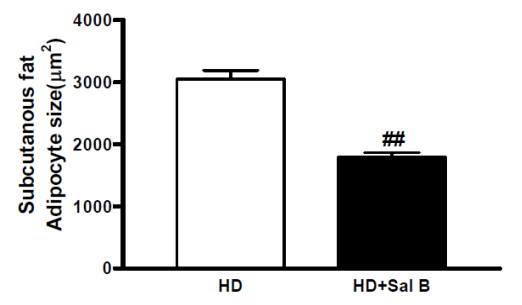

E

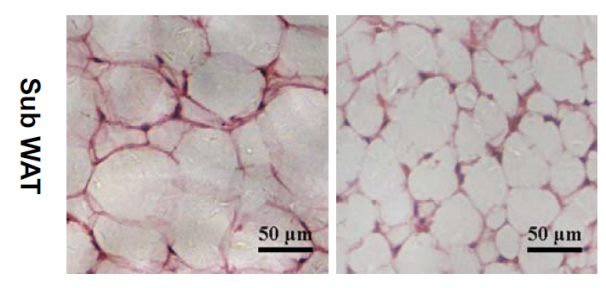

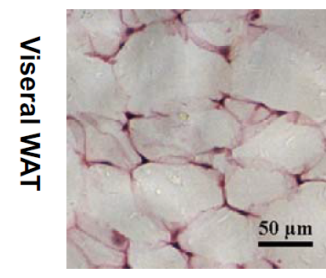

HD

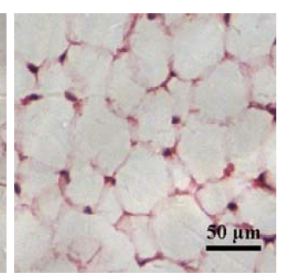

HD+Sal B

3C and 3D, administration of Sal B for 8 weeks not only improved insulin sensitivity but also lowered plasma glucose levels at $30 \mathrm{~min}, 60 \mathrm{~min}, 90 \mathrm{~min}$ and $120 \mathrm{~min}$ after glucose challenge compared to the control mice. These findings indicated that Sal B improved obesity-induced glucose intolerance and insulin sensitivity.

Sal B suppressed PPAR $\gamma-C / E B P \alpha$ and increased GATA 2/GATA 3 expression in high-fat dietinduced obese mice

A complex cascade of transcription factors controls adipogenesis. PPAR $\gamma$ and C/EBP $\alpha$ are at the center of this cascade [18]. A recent study showed that Sal B decreased glucocorticoid (GC)-induced adipogenic differentiation via downregulation of PPAR $\gamma$ mRNA expression [19]. To further understand the possible molecular mechanisms underlying the inhibition of adipogenesis by Sal B, we investigated the expression levels of several adipocyte marker proteins in visceral fat tissue by immunoblot. Eight weeks of Sal B administration depressed PPAR $\gamma$ and C/EBP $\alpha$ expression but increased GATA 2 and GATA 3 expression (Fig. 4A-D).

Sal B inhibited adipogenesis and decreased PPAR $\gamma$ and C/EBP $\alpha$ expression in 3T3-L1 preadipocytes

Next, we explored whether the effects of Sal B in vivo could be observed in vitro. 3T3L1 preadipocytes were cultured and induced to mature in the presence or absence of Sal 
Fig. 3. Salvianolic acid B improved glucose metabolism in high-fat diet-induced obese mice. A: Effect of Sal B on fasting blood glucose in high-fat diet-induced obese mice $(n=10)$. B: Sal B reduces the plasma insulin level of high-fat diet-induced obese mice $(n=10)$. C: IPITT of mice treated with a high-fat diet or $\mathrm{HD}+\mathrm{Sal}$ $\mathrm{B} ; \# P<0.05$, \#\#P<0.01 versus the HD group $(n=6)$. D: IPGTT ( $2 \mathrm{~g} / \mathrm{kg}$ ) and fasting blood glucose levels in mice treated with or without Sal B for 8 weeks. $\# P<0.05$, $\# \# P<0.01$ versus the HD group $(n=6)$. These data are represented as the means \pm SEM and were analyzed with Student's unpaired ttest.

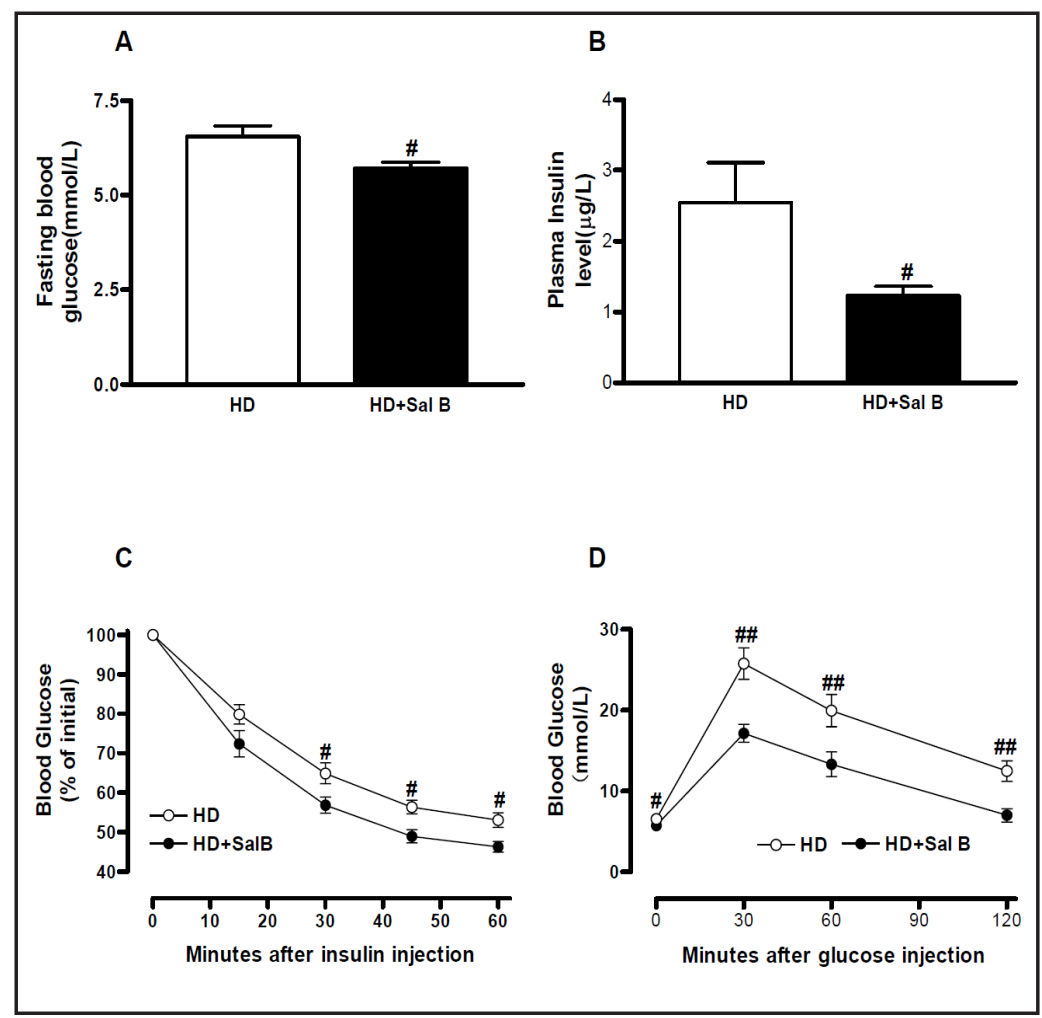

Fig. 4. Effects of salvianolic acid $\mathrm{B}$ on the protein expression of PPAR $\gamma, \mathrm{C} / \mathrm{EBP} \alpha$, GATA 2, and GATA 3 in obese mice. A-D: Effects of Sal $B$ on the protein expression of PPAR $\gamma, \mathrm{C} / \mathrm{EBP} \alpha$, GATA 2, and GATA 3 in high-fat dietinduced obese mice $(n=3)$. Protein expression is shown relative to GAPDH. $\# P<0.05$ versus the HD group $(n=3)$. The data are represented as the mean \pm SEM and were analyzed with Student's unpaired t-test.

A

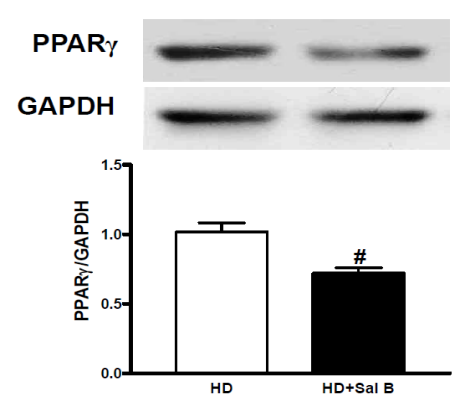

C

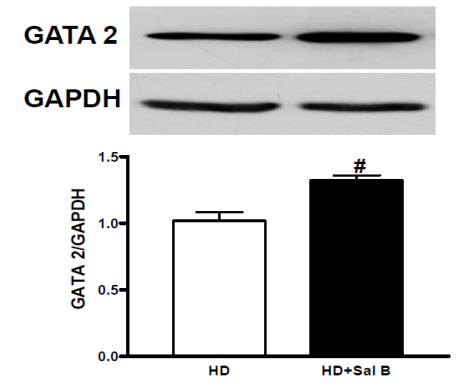

B
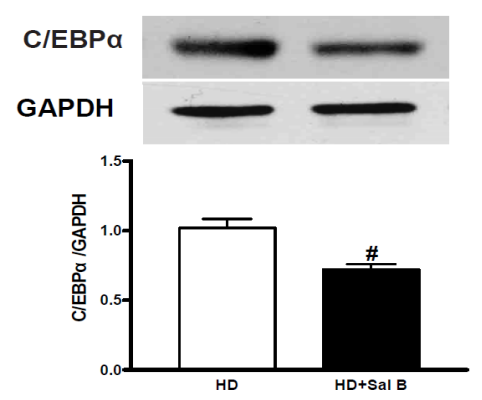

D

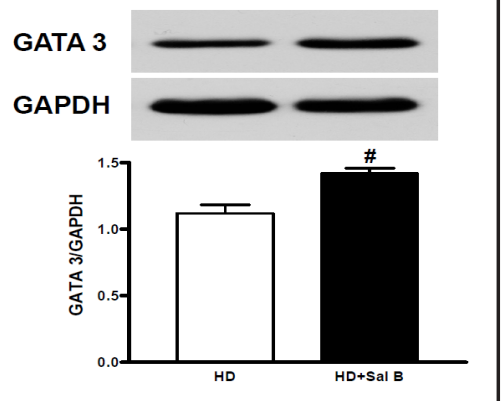

B. Sal B reduced the lipid droplets and the lipid content of adipocytes in a dose-dependent manner (Fig. 5A and B). Immunoblotting showed that Sal B (10 $\mu \mathrm{M})$ decreased PPAR $\gamma$ and $\mathrm{C} / \mathrm{EBP} \alpha$ expression in mature adipocytes but not in undifferentiated preadipocytes (Fig. 5C and D, Fig. 6A, C and D). Sal B increased GATA 2 and GATA 3 expression in both differentiated preadipocytes and undifferentiated preadipocytes (Fig. 5E and F, Fig. 6B, E and F). 


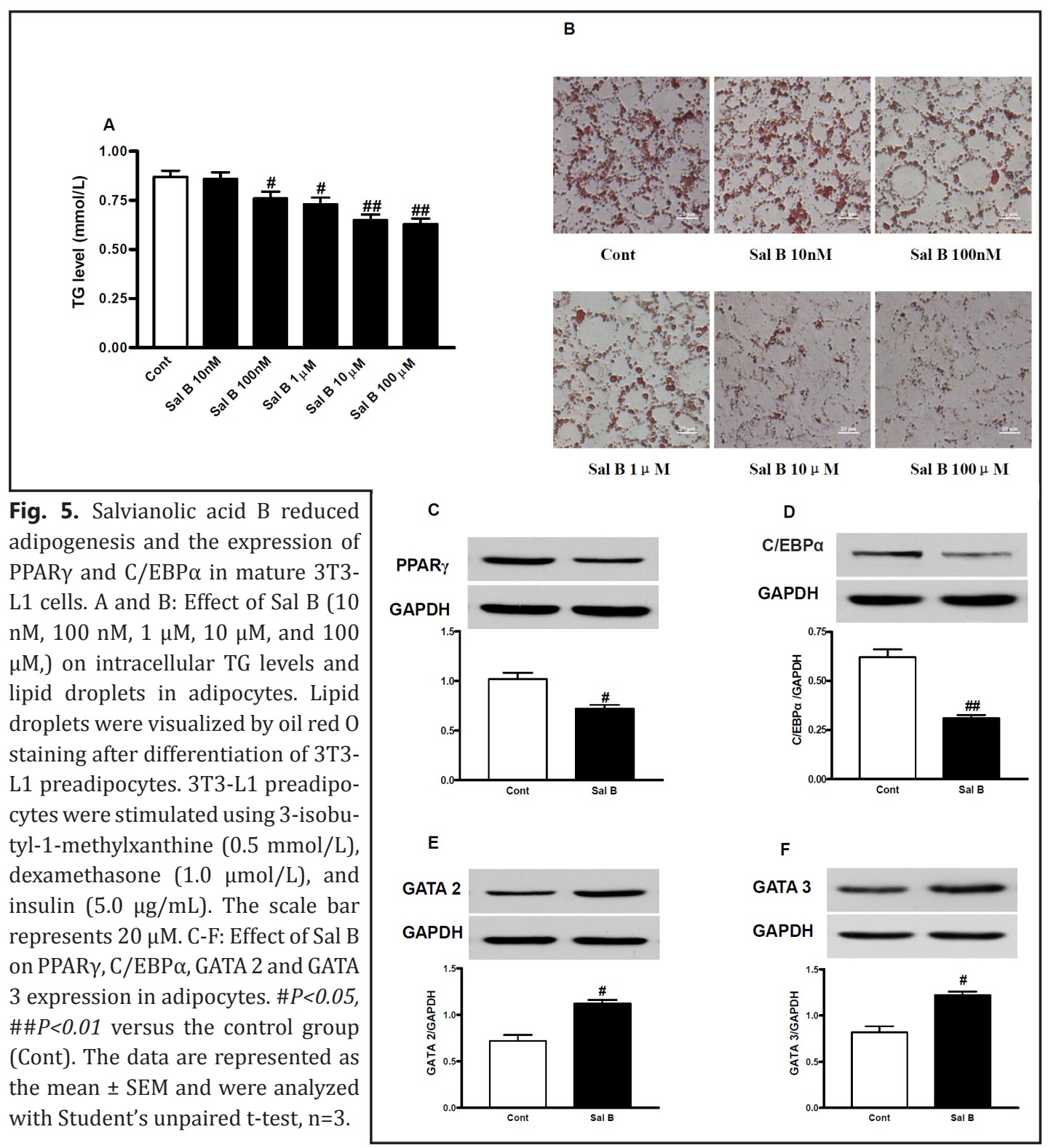

\section{Discussion}

The major findings of this study are that Sal B significantly reduced body weight and white adipose tissue weight, lowered lipid levels and improved insulin resistance in highfat diet-induced obese mice. Histological analysis indicated Sal B decreased adipocyte size in white adipose tissue. In mature, differentiated 3T3-L1 cells in vitro, Sal B decreased lipid content and the expression of PPAR $\gamma$ and C/EBP $\alpha$ while increasing GATA 2 and GATA 3 expression. This response was similar to that observed in the adipose tissue of obese mice in vivo.

Increasing evidence indicates that obese individuals have a substantially higher risk of developing many diseases such as type 2 diabetes, hyperlipidemia, and cardiovascular disease [20]. Thus, the search for compounds that can potentially aid in the treatment of obesity has intensified.

Studies have shown that Sal B has wide pharmacological effects such as increasing coronary blood flow, reducing excitability and conductivity of the myocardium, protecting 
Fig. 6. Effects of salvianolic acid B on the protein expression of PPAR $\gamma, \mathrm{C} /$ $\mathrm{EBP} \alpha$, GATA 2, and GATA 3 in preadipocytes. A-F: PPAR $\gamma, \mathrm{C} / \mathrm{EBP} \alpha$, GATA 2 and GATA 3 expression in 3T3-L1 preadipocytes with or without Sal B (10 $\mu \mathrm{M})$ stimulation. Protein expression is shown relative to GAPDH. $\# P<0.05$ versus the HD group $(n=3)$. The data are represented as the mean \pm SEM and were analyzed with Student's unpaired t-test.

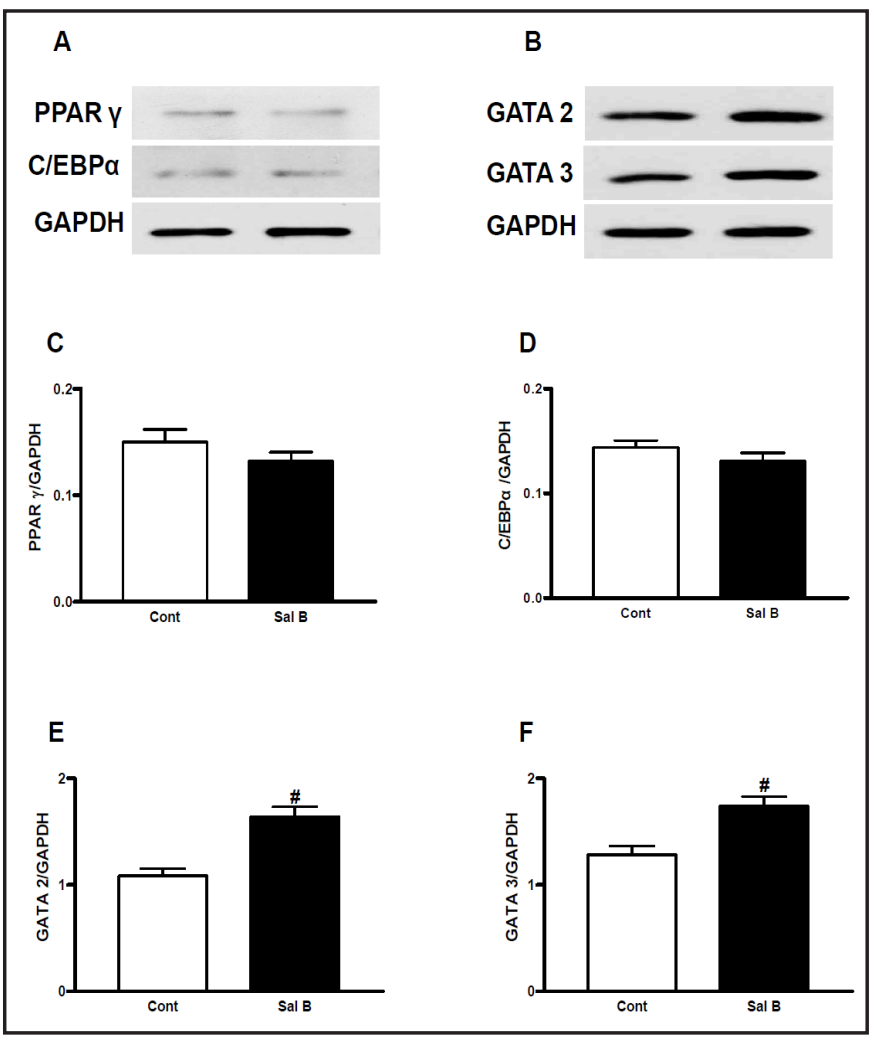

against myocardial ischemia/reperfusion (I/R) injury, improving microcirculation, inhibiting platelet aggregation and thrombosis, preventing atherosclerosis, protecting and improving kidney function, and reducing blood viscosity. Sal B also exhibits antibacterial, antiinflammatory and antioxidant properties and can protect brain tissue from I/R injury [7]. Previous studies demonstrated that the oral bioavailability of Sal B was $2.3 \%-3.9 \%$ in rats and approximately $1.07 \%$ in dogs [21-23]. After oral administration, salvianolic acid B was absorbed quickly with a $\mathrm{T}_{\max }$ of approximately $30 \mathrm{~min}$ [24]. Pretreatment with rifampicin, a representative inducer of drug-metabolizing enzymes and transporters, can increase the AUC and $\mathrm{C}_{\max }$ values and significantly decrease bile clearance values [25]. A recent study showed that Sal B prevented bone loss in glucocorticoid-treated rats through stimulation of osteogenesis and bone marrow angiogenesis and inhibition of adipogenesis. This study indicated that Sal B may have anti-obesity properties [19].

High-fat diet-treated C57BL/6J mice are a well known model of obesity and pre-type 2 diabetes that exhibit elevated blood glucose and impaired glucose tolerance [26]. They do not develop overt diabetes, but they do develop serious insulin resistance [26]. In this study, we first verified that Sal B not only attenuated weight gain but also decreased lipid levels in high-fat diet-induced obese mice.

Because adipocyte differentiation plays a major role in the process of fat mass growth [4], we then explored the effects of Sal B on visceral fat and subcutaneous fat. Our data indicated that Sal B decreased the weight of adipose tissue and the size of adipocytes, as determined by H\&E stain. In vitro, we used 3T3-L1 preadipocytes to explore the effects of Sal B on adipogenesis. The mouse-derived 3T3-L1 preadipocyte cell line is widely used for metabolic studies of obesity. Oil red O stain of mature differentiated 3T3-L1 cells indicated that Sal B reduced the accumulation of lipid droplets and decreased lipid content. These results indicated that Sal B may have an important role in the prevention of adipogenesis.

Weight loss is associated with a decrease in insulin concentration and an increase in insulin sensitivity [27]. We investigated whether the effects of Sal B on weight gain would have an impact on high-fat diet-induced insulin resistance. Eight weeks of Sal B administration by 
gavage improved obesity-induced insulin sensitivity and glucose intolerance, as measured by the IPITT and IPGTT, respectively.

PPAR $\gamma$ and C/EBP $\alpha$ are key activators of adipogenesis [28]. Recent reports showed that constitutive GATA 2 and GATA 3 expressions suppressed adipocyte differentiation and trapped cells at the preadipocyte stage and that this effect was mediated, at least in part, through the direct suppression of PPAR $\gamma$, both of which can form protein complexes with C/EBP $\alpha$ [29]. A recent study showed that Sal B stimulated bone marrow stromal cell (BMSC) differentiation into osteoblasts, increased osteoblast activity, and decreased GCassociated adipogenic differentiation by downregulation of PPAR $\gamma$ mRNA expression [19]. We investigated the effects of Sal B on the expression of these proteins in the visceral fat tissue of high-fat diet-induced obese mice. Immunoblotting showed that administration of Sal B decreased the expression of PPAR $\gamma$ and C/EBP $\alpha$ and increased GATA 2 and GATA 3 expression.

PPAR $\gamma$ is a ligand-activated transcription factor and functions as a heterodimer with retinoid X receptor (RXR) [30]. Activation of PPAR $\gamma$ by thiazolidinediones can reduce insulin resistance and hyperglycemia in patients with type 2 diabetes; however, these drugs can also cause weight gain [31]. It has been shown that moderate reduction of PPAR $\gamma$ activity in mice prevented the insulin resistance and obesity induced by a high-fat diet [32]. In this study, PPAR $\gamma$ antagonists decreased TG content in white adipose tissue, skeletal muscle, and liver [32]. These inhibitors potentiated the effects of leptin and increased fatty acid combustion and energy dissipation, thereby ameliorating high-fat diet-induced obesity and insulin resistance [32]. These results indicated that appropriate functional antagonism of PPAR $\gamma$ may be a logical approach for protection against obesity and obesity-related diseases.

Our study indicated that Sal B increased GATA 2 and GATA 3 expression while it suppressed PPAR $\gamma$ and $\mathrm{C} / \mathrm{EBP} \alpha$ expression. Sal B prevented the differentiation of preadipocytes and reduced weight gain in obese mice. However, the specific mechanisms by which Sal B prevents the differentiation of preadipocytes require further investigation.

In summary, our findings provided the first evidence that Sal B treatment may be an effective therapeutic strategy for attenuating weight gain in obese individuals.

\section{Acknowledgments}

This work was supported by the National Natural Science Foundation of China (NO. 81373940), the Specialized Research Fund for the Doctoral Program of Higher Education (NO. 20133519120001) and the Provincial Natural Science Foundation of Fujian (NO. 2012J05152 and 2012Y4005); the Scientific Research Fund of Chengdu Medical College, China (NO.CYZ13-001) and the Scientific Research Fundation of the Education Department of Sichuan Province, China (N0.14ZB0234).

\section{Disclosure Statement}

None

\section{References}

1 Brantley PJ, Myers VH, Roy HJ: Environmental and lifestyle influences on obesity. J La State Med Soc 2005;157:S19-27.

2 Steinberger J, Daniels SR: Obesity, insulin resistance, diabetes, and cardiovascular risk in children: An american heart association scientific statement from the atherosclerosis, hypertension, and obesity in the young committee (council on cardiovascular disease in the young) and the diabetes committee (council on nutrition, physical activity, and metabolism). Circulation 2003;107:1448-1453. 
- 3 Hu Y, Davies GE: Berberine inhibits adipogenesis in high-fat diet-induced obesity mice. Fitoterapia 2010;81:358-366.

- Z Zhou YT, Wang ZW, Higa M, Newgard CB, Unger RH: Reversing adipocyte differentiation: Implications for treatment of obesity. Proc Natl Acad Sci U S A 1999;96:2391-2395.

-5 Dubois SG, Heilbronn LK, Smith SR, Albu JB, Kelley DE, Ravussin E: Decreased expression of adipogenic genes in obese subjects with type 2 diabetes. Obesity (Silver Spring) 2006;14:1543-1552.

-6 Tong Q, Tsai J, Tan G, Dalgin G, Hotamisligil GS: Interaction between gata and the c/ebp family of transcription factors is critical in gata-mediated suppression of adipocyte differentiation. Mol Cell Biol 2005;25:706-715.

7 Wang J, Xiong X, Feng B: Cardiovascular effects of salvianolic acid b. Evid Based Complement Alternat Med 2013;2013:247948.

8 Shou Q, Pan Y, Xu X, Xu J, Wang D, Ling Y, Chen M: Salvianolic acid b possesses vasodilation potential through no and its related signals in rabbit thoracic aortic rings. Eur J Pharmacol 2012;697:81-87.

-9 Du G, Zhu H, Yu P, Wang H, He J, Ye L, Fu F, Zhang J, Tian J: Smnd-309 promotes angiogenesis in human umbilical vein endothelial cells through activating erythropoietin receptor/stat3/vegf pathways. Eur J Pharmacol 2013;700:173-180.

10 Pan CH, Chen CW, Sheu MJ, Wu CH: Salvianolic acid b inhibits sdf-1alpha-stimulated cell proliferation and migration of vascular smooth muscle cells by suppressing cxcr4 receptor. Vascul Pharmacol 2012;56:98105.

11 Yang TL, Lin FY, Chen YH, Chiu JJ, Shiao MS, Tsai CS, Lin SJ, Chen YL: Salvianolic acid b inhibits low-density lipoprotein oxidation and neointimal hyperplasia in endothelium-denuded hypercholesterolaemic rabbits. J Sci Food Agric 2010;91:134-141.

12 Sun A, Liu H, Wang S, Shi D, Xu L, Cheng Y, Wang K, Chen K, Zou Y, Ge J: Salvianolic acid b suppresses maturation of human monocyte-derived dendritic cells by activating ppargamma. Br J Pharmacol 2011;164:2042-2053.

13 Wang P, Yan Z, Zhong J, Chen J, Ni Y, Li L, Ma L, Zhao Z, Liu D, Zhu Z: Transient receptor potential vanilloid 1 activation enhances gut glucagon-like peptide- 1 secretion and improves glucose homeostasis. Diabetes 2012;61:2155-2165.

14 Kang JH, Goto T, Han IS, Kawada T, Kim YM, Yu R: Dietary capsaicin reduces obesity-induced insulin resistance and hepatic steatosis in obese mice fed a high-fat diet. Obesity (Silver Spring) 2010;18:780-787.

15 Yan Z, Ni Y, Wang P, Chen J, He H, Sun J, Cao T, Zhao Z, Luo Z, Chen L, Liu D, Zhu Z: Peroxisome proliferatoractivated receptor delta protects against obesity-related glomerulopathy through the p38 mapk pathway. Obesity (Silver Spring) 2013;21:538-545.

16 Zhang LL, Yan Liu D, Ma LQ, Luo ZD, Cao TB, Zhong J, Yan ZC, Wang LJ, Zhao ZG, Zhu SJ, Schrader M, Thilo F, Zhu ZM, Tepel M: Activation of transient receptor potential vanilloid type-1 channel prevents adipogenesis and obesity. Circ Res 2007;100:1063-1070.

17 Ros Perez M, Medina-Gomez G: [obesity, adipogenesis and insulin resistance]. Endocrinol Nutr 2011;58:360-369.

18 Rosen ED, Hsu CH, Wang X, Sakai S, Freeman MW, Gonzalez FJ, Spiegelman BM: C/ebpalpha induces adipogenesis through ppargamma: A unified pathway. Genes Dev 2002;16:22-26.

19 Cui L, Li T, Liu Y, Zhou L, Li P, Xu B, Huang L, Chen Y, Tian X, Jee WS, Wu T: Salvianolic acid b prevents bone loss in prednisone-treated rats through stimulation of osteogenesis and bone marrow angiogenesis. PLoS One 2012; 7:e34647.

20 Goran MI, Ball GD, Cruz ML: Obesity and risk of type 2 diabetes and cardiovascular disease in children and adolescents. J Clin Endocrinol Metab 2003;88:1417-1427.

21 Wu YT, Chen YF, Hsieh YJ, Jaw I, Shiao MS, Tsai TH: Bioavailability of salvianolic acid b in conscious and freely moving rats. Int J Pharm 2006;326:25-31.

22 Zhou L, Chow MS, Zuo Z: Effect of sodium caprate on the oral absorptions of danshensu and salvianolic acid b. Int J Pharm 2009;379:109-118.

23 Gao DY, Han LM, Zhang LH, Fang XL, Wang JX: Bioavailability of salvianolic acid b and effect on blood viscosities after oral administration of salvianolic acids in beagle dogs. Arch Pharm Res 2009;32:773-779.

24 Peng Q, Gong T, Zuo J, Liu J, Zhao D, Zhang Z: Enhanced oral bioavailability of salvianolic acid b by phospholipid complex loaded nanoparticles. Pharmazie 2008;63:661-666. 
25 Zhao D, Gao ZD, Han DE, Li N, Zhang YJ, Lu Y, Li TT, Chen XJ: Influence of rifampicin on the pharmacokinetics of salvianolic acid b may involve inhibition of organic anion transporting polypeptide (oatp) mediated influx. Phytother Res 2011;26:118-121.

26 Buettner R, Scholmerich J, Bollheimer LC: High-fat diets: Modeling the metabolic disorders of human obesity in rodents. Obesity (Silver Spring) 2007;15:798-808.

27 Kopp HP, Kopp CW, Festa A, Krzyzanowska K, Kriwanek S, Minar E, Roka R, Schernthaner G: Impact of weight loss on inflammatory proteins and their association with the insulin resistance syndrome in morbidly obese patients. Arterioscler Thromb Vasc Biol 2003;23:1042-1047. Farmer SR: Transcriptional control of adipocyte formation. Cell Metab 2006;4:263-273.

29 Tong Q, Dalgin G, Xu H, Ting CN, Leiden JM, Hotamisligil GS: Function of gata transcription factors in preadipocyte-adipocyte transition. Science 2000;290:134-138.

30 Abdelrahman M, Sivarajah A, Thiemermann C: Beneficial effects of ppar-gamma ligands in ischemiareperfusion injury, inflammation and shock. Cardiovasc Res 2005;65:772-781.

31 Bloomgarden ZT: Thiazolidinediones. Diabetes Care 2005;28:488-493.

32 Yamauchi T, Waki H, Kamon J, Murakami K, Motojima K, Komeda K, Miki H, Kubota N, Terauchi Y, Tsuchida A, Tsuboyama-Kasaoka N, Yamauchi N, Ide T, Hori W, Kato S, Fukayama M, Akanuma Y, Ezaki O, Itai A, Nagai R, Kimura S, Tobe K, Kagechika H, Shudo K, Kadowaki T: Inhibition of rxr and ppargamma ameliorates dietinduced obesity and type 2 diabetes. J Clin Invest 2001;108:1001-1013. 